\title{
Formation of multiple-oocyte follicles in culture
}

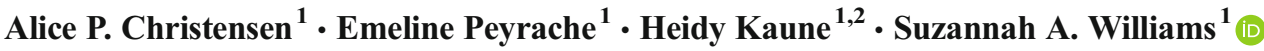

Received: 18 November 2016 / Accepted: 31 May 2017 / Published online: 22 September 2017 / Editor: Tetsuji Okamoto

(C) The Author(s) 2017. This article is an open access publication

\begin{abstract}
Basement membranes are found in every organ of the body. They provide structure and a selective filter for molecules. The ovary is no different with the follicular basal lamina (FBL) separating the granulosa and theca cells, facilitating regulation of the changing follicular environment providing appropriate conditions for the developing oocyte. The FBL is modified in Clgalt1 Mutant mice (C1galt1 ${ }^{\mathrm{FF}}:$ ZP3Cre) resulting from oocyte-specific deletion of Clgalt1. Changes in the FBL lead to follicles joining to generate multiple-oocyte follicles (MOFs); where two or more oocytes are contained within a single follicle. This study aimed to determine if single-oocyte follicles could join in culture to become MOFs by co-culturing preantral follicles from Control or Mutant mice. Co-cultured follicles from both Control and Mutant follicles could superficially fuse ( $73 \%$ of Control follicle pairs; $84 \%$ of Mutant). Confocal microscopy revealed alterations in the organization of the space between follicles but was unable to discern MOFs. When co-cultured follicle pairs were embedded, sectioned and stained with haematoxylin, it was revealed that MOFs had formed from 50\% of Mutant follicle pairs but none from Control follicle pairs. In conclusion, MOFs can form from Clgalt1 Mutant follicles in culture and this model is a useful tool to elucidate the role of the oocyte in follicle development and the generation and function of the FBL. Furthermore, understanding the relationship between oocyte function and FBL generation will likely
\end{abstract}

Suzannah A. Williams

suzannah.williams@obs-gyn.ox.ac.uk

1 Nuffield Department of Obstetrics and Gynaecology, University of Oxford, Women's Centre, Level 3, John Radcliffe Hospital, Oxford OX3 9DU, UK

2 Centre for Biomedical Research, Facultad de Medicina, Universidad Diego Portales, 8370068 Santiago, Chile provide insight into optimizing conditions for follicle culture, which is important for fertility treatments and ART.

Keywords Ovary · Extracellular matrix · Core $1 O$-glycans * C1galt1 · Confocal microscopy

\section{Introduction}

The ovary has evolved numerous functions to facilitate the development and ovulation of an egg. To achieve this, granulosa, theca cells, and oocytes form follicle units within the ovary with basal lamina (BL) separating the granulosa and theca cells compartments. Signalling between all three of these cell types is essential to support follicle development, oocyte development, and ovulation.

The BL, such as that separating the granulosa and theca cells, is a barrier found in all organs of the body. It acts as a gateway for selective molecule passage, and as a structure to support and define boundaries within a tissue. The follicle basal lamina (FBL) is comprised of extracellular matrix molecules such as collagen IV, laminin, nidogen, and perlecan (Yurchenco and Patton 2009). Genetically inherited or acquired alterations of the BL have been linked to complex diseases such as cancer and diabetes. Cancer cells migrate along collagen fibres of the extracellular matrix (Condeelis and Segall 2003; Fein and Egeblad 2013) and degrade the basement membrane by invadopodia (Cmoch et al. 2014) allowing local invasion of cancer cells and metastatic spread. In type 2 diabetes, thickening and remodelling of the BL occurs (Siperstein et al. 1968; Hayden et al. 2005). The body's response to this deposition contributes to the secondary complications of diabetes in cardiovascular and renal systems (Hayden et al. 2005). 
In the ovary, the FBL regulates the environment within the follicle during development, and acts as a structural barrier between the vascular theca cells and avascular granulosa cells (Anderson and Spielman 1971; Christensen et al. 2015). This discriminatory divider is comprised mainly of collagen and laminin (Rodgers et al. 1999; Berkholtz et al. 2006) and allows only molecules of a certain size and charge to cross (Hess et al. 1998). The FBL changes with the developing follicle, adapting its permeability and constituent molecules. The FBL has several different morphologies, e.g., thick and loopy, and these different morphologies have been linked to the developmental potential of the oocyte (Irving-Rodgers et al. 2009; Rodgers and Irving-Rodgers 2010).

Interestingly, the oocyte itself has recently been shown to have a role in the generation of the FBL (Christensen et al. 2015; Grasa et al. 2015). Oocytes from mice with an oocytespecific deletion of Clgalt1 (previously known as T-syn) (Williams et al. 2007) do not produce the enzyme core 1 $\beta 1,3$-galactosyltransferase 1 (also known as T-synthase) and therefore cannot form core 1-derived $O$-glycans (Ju et al. 2002a, b). The absence of these glycans in the oocyte leads to follicles with a structurally modified FBL with changes in laminin and collagen distribution (Christensen et al. 2015; Grasa et al. 2015). Oocyte-specific Clgalt1 Mutant mice have a phenotype of increased fertility (Williams et al. 2007), modified cumulus expansion (Ploutarchou et al. 2015) and ovaries with increased numbers of multiple-oocyte follicles (MOFs); where two or more oocytes are contained within a FBL (Williams et al. 2007). However, it is important to note that the increased fertility does not result from the MOFs ovulating multiple eggs since the preovulatory MOFs do not contain multiple healthy eggs (Christensen et al. 2015) and the number of corpora lutea is equal to the ovulation rate (Williams and Stanley 2008). MOFs can occur as a natural phenomenon from incomplete germ cell nest breakdown (Pepling 2012), which happens just after birth in differing degrees in different strains of wildtype mice. In the Clgalt1 Mutant mice, a novel mechanism of MOF formation occurs. We can conclude this because (i) Clgalt1 deletion occurs after follicle nest breakdown and (ii) MOFs are more numerous at later stages of follicle development (Williams et al. 2007).

Follicle culture allows follicle development to be studied in a controlled in vitro environment enabling specific functions to be assessed. Follicle co-culture is where multiple follicles are placed together in the same culture well allowing signalling and physical interaction. Survival has been improved by the co-culture of five or ten mouse primary and secondary follicles (Hornick et al. 2013). However, co-culture of two preantral mouse follicles did not demonstrate altered growth when compared to follicles cultured alone (Spears et al. 1996). Therefore, although follicles can interact in culture, the intricacies of these interactions are complex. Migration of theca cells between co-cultured follicles has also been observed
(Campbell et al. 2013). Such cellular migration might be contributing to the formation of MOFs in Clgalt1 Mutants in vivo since morphological changes in the theca cells of these mice have recently been demonstrated (Christensen et al. 2015). Co-culture of wildtype mouse follicles do not form MOFs in vitro (Spears et al. 1996).

This paper aims to determine if follicles can join together in vitro to generate MOFs by co-culturing follicles from Clgalt1 Mutant mice.

\section{Materials and methods}

Mice All of the studies using mice (Mus musculus) were implemented with approval of the Local Ethical Review Panel at the University of Oxford under licence in accordance with the UK Animals (Scientific Procedures) Act 1986. Mutant mice were homozygous $C$ lgalt $^{\mathrm{F} / \mathrm{F}}$ carrying a $\mathrm{ZP} 3$ Cre recombinase transgene and Control mice were homozygous for $\mathrm{Clgalt}^{\mathrm{F} / \mathrm{F}}$ on a mixed background $(129 / \mathrm{SvlmJ}$ and C57BL/6 J) (Williams et al. 2007). The ZP3 promotor is only active in oocytes from the primary stage of follicle development onwards (Philpott et al. 1987) and the ZP3Cre recombinase transgene has no impact on fertility (Shi et al. 2004; Williams et al. 2007).

Genotyping Genotyping was carried out using ear or tail genomic DNA adapted from (Williams et al. 2007; Grasa et al. 2015) as follows. Every $25 \mu \mathrm{l}$ PCR reaction had $2.5 \mu \mathrm{l}$ of $10 \mathrm{x}$ PCR buffer (Bioline, London, UK), $0.75 \mu \mathrm{l}$ of $50 \mathrm{mM}$ magnesium chloride solution (Bioline, London, UK), $0.5 \mu \mathrm{l}$ of DNTP (Roche, Mannheim, Germany), $0.5 \mu \mathrm{l}$ of each primer (Eurogentec, Liege Science Park, Belgium), $1 \mu$ l of genomic DNA (ear or tail), and either $0.5 \mu \mathrm{l}$ of Taq polymerase (Bioline) to identify Cre transgene, or $0.15 \mu \mathrm{l}$ of Taq polymerase to identify the floxed Clgalt1. Primers TS1 (Williams et al. 2007) and TS8 (Christensen et al. 2015) or FB33 and FB34 (Batista et al. 2012) were used to identify the floxed Clgalt1 allele. Primers PS502 and PS607 were used to identify the Cre transgene (Shi et al. 2004).

Follicle culture Ovaries from 6-week Control and Mutant mice were dissected using a Leica M125 microscope (Leica Microsystems, Germany) and placed in warm Leibovitz L-15 culture medium (Gibco; Fisher Scientific, Loughborough, Leicestershire) supplemented with $3 \mathrm{mg} / \mathrm{ml}$ of bovine serum albumin (Sigma-Aldrich, Gillingham, Dorset). Individual preantral follicles were dissected manually using acupuncture needles. Follicles with a central oocyte, intact FBL, surrounded by theca cells and a follicle diameter between 120 to $170 \mu \mathrm{m}$, were selected for further culture. Each follicle was placed in an untreated 96-well plate (Nunc, Fisher Scientific) and cultured individually for $24 \mathrm{~h}$ at $37{ }^{\circ} \mathrm{C}$ with 
$5 \% \mathrm{CO}_{2}$ in pre-equilibriated Alpha Minimum Essential Medium ( $\alpha$-MEM, Gibco; Fisher Scientific) supplemented with $5 \%$ foetal bovine serum (Labtech, Uckfield, UK), 5 IU/ $\mathrm{ml}$ of recombinant human FSH (r-hFSH, Gonal-F, Merk Serono, Feltham, UK), with $0.5 \%$ ascorbic acid (Fisher Scientific) and vital stain neutral red (Sigma Aldrich). After $24 \mathrm{~h}$ in culture, follicles were transferred to a new well in pairs (term used to describe two follicles co-cultured together) with fresh pre-equilibrated culture medium. Thereafter, $50 \%$ of culture medium was replaced with fresh pre-equilibrated culture medium daily. Follicle pairs were cultured for 4 or $5 \mathrm{~d}$ and images collected daily using a MicroPublisher 5-ORTU QImagine camera. Follicle pairs were classified as dead if one or both follicles had died. At the end of the culture period, healthy follicle pairs that were superficially fused (SF) were washed in Phosphate Buffered Saline (PBS, pH 7.2-7.4) at $37^{\circ} \mathrm{C}$ for $10 \mathrm{~min}$ and permeabilized with $0.5 \%$ Triton $\mathrm{X}-100$ (Sigma-Aldrich) and $0.25 \%$ paraformaldehyde in PBS at $37^{\circ} \mathrm{C}$ for $40 \mathrm{~min}$. Follicle were then fixed with $4 \%$ paraformaldehyde at room temperature for $30 \mathrm{~min}$, followed by two washes in PBS for 10 min and stored in PBS/0.02\% sodium azide (Sigma-Aldrich) at $4{ }^{\circ} \mathrm{C}$ until stained for confocal microscopy.

Follicle staining for confocal microscopy Fixed follicle pairs were washed twice in PBS for 5 min each, incubated in $0.1 \%$ Triton X-100 in PBS for $30 \mathrm{~min}$ and washed twice in PBS for $5 \mathrm{~min}$ each. Follicles were stained for $2 \mathrm{~h}$ with $0.01 \%$ Atto $647 \mathrm{~N}-$ Phalloidin (Sigma-Aldrich) and $2.5 \mu \mathrm{g} / \mathrm{ml}$ propidium iodide (Sigma-Aldrich) with $0.1 \%$ Tween 20 (Fisher Scientific) in PBS at $4{ }^{\circ} \mathrm{C}$ in the dark and then washed twice in PBS for $10 \mathrm{~min}$ each. Follicles were mounting in Vectashield (Vectorlabs, Peterborough, UK) on slides using secure seal spacers (Fisher Scientific) to create a cavity and avoid compression. Slides were stored at $4{ }^{\circ} \mathrm{C}$ until microscopy analysis.

Confocal microscopy Follicle pairs (selected at random from follicle pairs that appeared healthy on day 6 of culture) were examined using an inverted confocal microscope (Zeiss). Two channels were used to produce true-colour RGB images. The scans were performed separately due to the excessive proximity of the wavelength of Ph and PI (respectively $644 \mathrm{~nm}$ and $617 \mathrm{~nm}$ ). Acquisition and processing of images was carried out using ZEN imaging software (Zeiss, Germany). Serial optical sections of $7 \mu \mathrm{m}$ thickness were carried out and individual images per channel acquired for all optical sections. Images were then merged to reveal co-localization of staining (mounted follicles were returned to $4{ }^{\circ} \mathrm{C}$ ).

Histological processing and analysis Follicle pairs (selected at random from follicle pairs that appeared healthy on day 6 of culture) were analysed with confocal microscopy were processed for histological analysis. Follicle pairs were removed from Vectashield and washed ten times in PBS for 3 min each before dehydrating through increasing concentrations of ethanol $(\mathrm{EtOH})$ at $70 \%, 80 \%$, and $95 \%$ (twice for $10 \mathrm{~min}$ each), $100 \% \mathrm{EtOH}$ (four times for $10 \mathrm{~min}$ each). Follicle pairs were then incubated in xylene (twice for $3 \mathrm{~min}$ and once for $4 \mathrm{~min}$ ) before embedding in paraffin wax $\left(72{ }^{\circ} \mathrm{C}\right.$ for $1 \mathrm{~h}$ before setting at room temperature). Serial sections of $3 \mu \mathrm{m}$ were collected onto glass slides and heated for $1 \mathrm{~h}$ at $62{ }^{\circ} \mathrm{C}$.

Sections were dewaxed in xylene ( 3 times for $4 \mathrm{~min}$ ) and rehydrated by immersion in decreasing concentrations of $\mathrm{EtOH}$ at $100 \%$ (twice), $90 \%, 70 \%$, and $50 \%$ for $1 \mathrm{~min}$. Sections were washed three times $1 \mathrm{~min}$ with nanopure water and stained with Haematoxylin (Thermo Scientific) for $5 \mathrm{~min}$. Sections were then dehydrated through increasing concentrations of ethanol, xylene and mounted using Depex. Sections were evaluated with a microscope (Leica DM 2500) and images collected.

Analysis Statistical analysis was carried out using Graphpad Prism 5 (GraphPad Software Inc., San Diego, CA). Fishers test was used to analyse differences in the number of SF follicles between control and mutant and the survival rate of follicle pairs in culture; follicle pair survival included both $\mathrm{SF}$ and non-SF follicles.

\section{Results}

Mutant and Control pairs of co-cultured follicles can superficially fuse during in vitro culture Follicle pairs (two follicles of the same genotype cultured in the same well) were cultured for $5 \mathrm{~d}$ and evaluated daily. Throughout the culture period some follicle pairs remained as separate units Fig. $1 a$ while other follicles were in contact with each other; we describe this as superficially fused (SF) Fig. $1 b$. After 5 days of co-culture, $73 \%$ of Control follicle pairs and $84 \%$ of Mutant follicle pairs were SF Fig. 1c. Most of the superficial fusing happened early in the culture; by day 2, $62 \%$ of Control and $60 \%$ of Mutant pairs had SF. Follicles that were SF did not separate later in culture.

Gross morphology assessed during in vitro culture did not reveal if follicle pairs were MOFs Although co-cultured follicles became SF during in vitro culture, it remained unclear whether the FBL had been breached and a MOF generated. However, this gross visual analysis did reveal differences between the pairs. In some follicle pairs, one follicle became dominant while the subordinate follicle did not appear to be dead or dying Fig. $2 a-e$, while in other pairs, both follicles remained equivalent in size Fig. $2 f-j$. 
Figure 1. Follicles in co-culture can superficially fuse (SF). (a) Image of a follicle pair that remained separated during culture. (b) Image of a follicle pair that are SF. (c) There is no difference in the percentage of SF follicle pairs between Control and Mutant at the end of culture (Control mice $n=6$, follicle pairs $n=26$ with 19 SF; Mutant mice $n=5$, follicle pairs $n=25$ with 21 $\mathrm{SF})$.
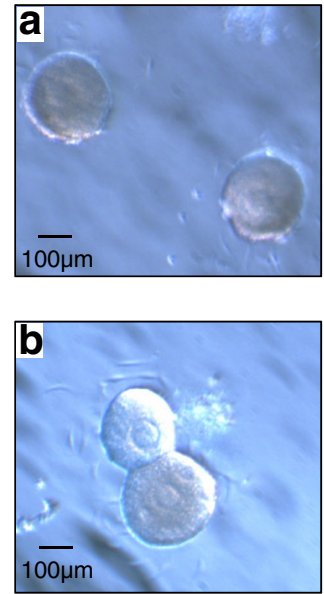

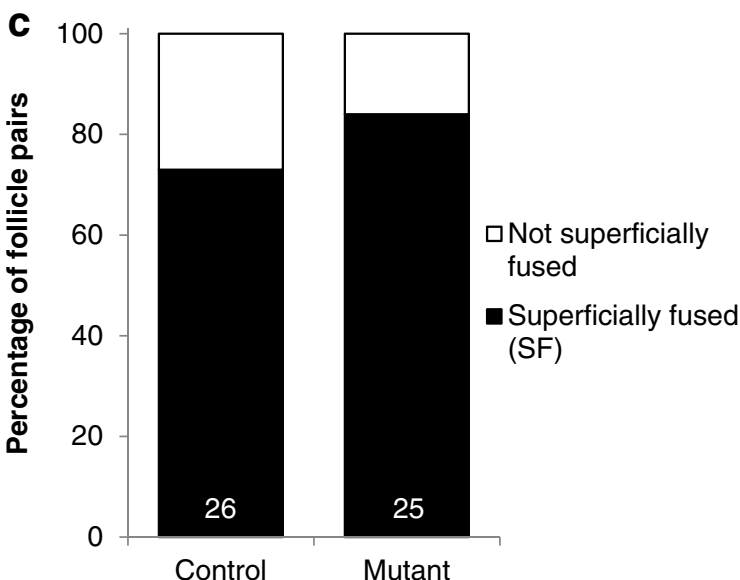

Mutant follicles survival in culture compared to Control follicles Follicle pair survival was determined on each day of follicle culture. Mutant follicle pairs had equivalent survival compared to Control follicle pairs after $6 \mathrm{~d}$ of follicle culture. (Fig. 3).

Confocal microscopy of SF follicle pairs revealed alterations in the joining between the follicle pairs but was not able to ascertain if MOFs formed Follicle pairs were analysed with confocal imaging to determine if MOFs could be identified (Control mice $n=4$ and $n=4$ follicle pairs, Mutant mice $n=4$ and $n=9$ follicle pairs). Alterations in the FBL can be seen with confocal microscopy. Confocal images were analysed for MOF formation by assessing the area where co-cultured follicles were touching. In some follicles pairs, although they appeared SF in culture, on confocal imaging the two follicles were clearly separate with a defined FBL Fig. $4 a-d$. Where the follicle pairs meet was either a clear separation between the granulosa and the theca layers Fig. $4 a^{\prime}-d^{\prime}$, or a potential breach in the FBL Fig. $4 e-h$. This is where an indistinct border between the follicles is revealed Fig. $4 e^{\prime}-h$ '. However, it is unclear using confocal microscopy whether a complete breakdown in the FBL occurred, to form a MOF, or if the FBL remained intact between follicles.

Mutant preantral follicles are able to form multiple-oocyte follicles (MOFs) when co-cultured in vitro After confocal imaging, cultured follicle pairs were fixed, embedded, sectioned and stained with haematoxylin (Control mice $n=3$ and $n=3$ follicle pairs, Mutant mice $n=4$ and 4 follicle pairs). Analysis of sequential sections stained with haematoxylin was previously used to assess FBL and MOF formation (Williams and Stanley 2008). The FBL of follicle pairs was analysed and a MOF was confirmed if there was a breach of the FBL between the follicles in three or more sequential sections. Using this analysis, we were able to confidently conclude that Mutant follicle pairs formed MOFs (Table 1), whereas no Control follicle pairs formed MOFs.

In Control follicle pairs, follicles remained as single-oocyte follicles (SOFs) and a clear FBL between the two follicles was observed through all of the serial sections Fig. $5 a-f$ and $a^{\prime}-f$ '. A clear FBL was also observed in most sections of the Mutant follicle pairs Fig. $5 g, l, g^{\prime}$ and $l$; however in some sections, a gap in the FBL between follicles was observed, consistent with MOF formation Fig. $5 h-k$ and $h^{\prime}-k$ '.
Figure 2. Images of superficially fused (SF) follicles on each day of co-culture. Images of the same follicle pair taken each day during the 5 days of follicle culture. $(a-e)$ This follicle pair is SF with one follicle becoming dominant. $(f-j)$ The follicle pair is SF and both follicles remain even in size throughout the culture period. It is not possible to discern if follicle pairs that had SF had formed MOFs.

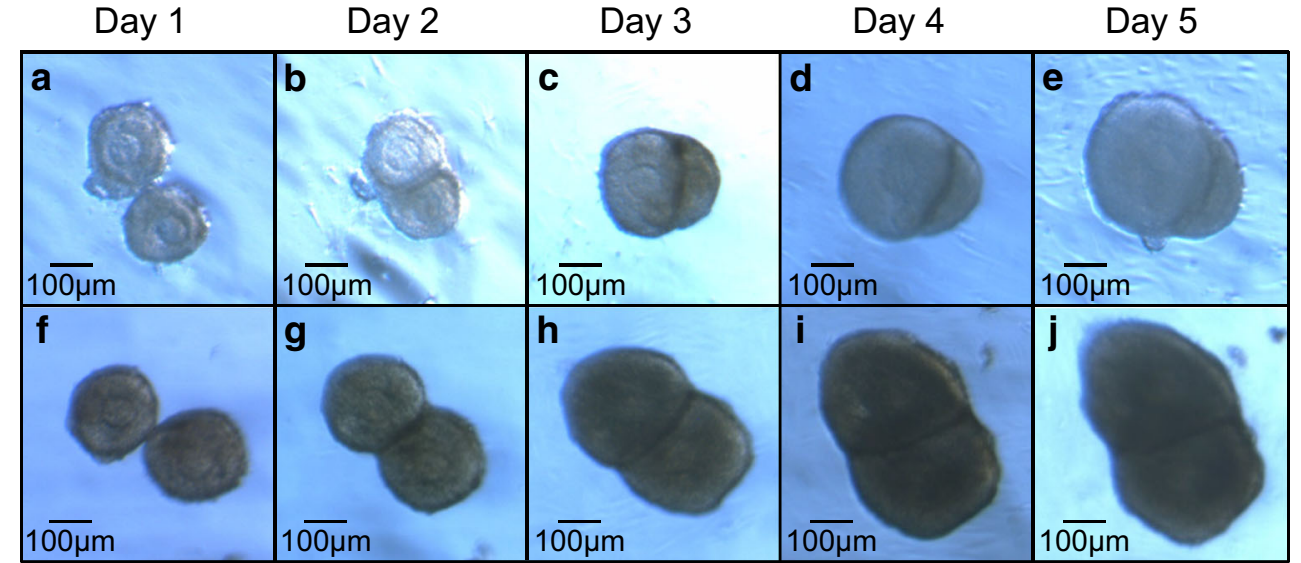




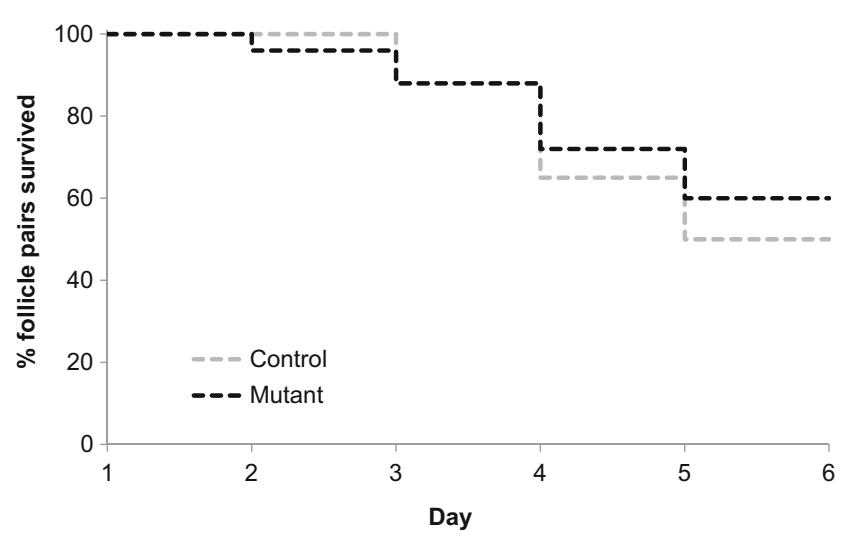

Figure 3. Survival rate on each $\mathrm{d}$ of follicle culture for Control and Mutant follicle pairs (Control $n=26$ follicle pairs from $n=6$ mice; Mutant $n=25$ follicle pairs from $n=5$ mice).

\section{Discussion}

Multiple-oocyte follicles that form though the fusion of adjacent FBLs exist in Clgalt1 Mutant ovaries (Williams and Stanley 2008). The aim of this study was to determine if FBL fusion and MOF formation can occur during co-culture of preantral follicles in vitro. In this study, we have demonstrated that follicles from Clgalt1 Mutant mice can join to form MOFs in vitro as well as in vivo (Williams and Stanley 2008). Follicles from Control mutant mice, in line with follicles from wild-type mice (Spears et al. 1996), do not form MOFs in culture. However, analysis of gross morphology of the follicles during culture and confocal microscopy are not reliable methods to confirm MOF formation. MOFs are generated in the Clgalt1 Mutant mouse as a result of oocytes lacking core $1 O$-glycans and thus MOF development in culture demonstrates that the environment of the ovary, beyond that of the follicle itself, is not required for MOF formation. Identifying that follicles can join in vitro provides a useful tool for further research particularly to unravel how the oocyte regulates the form and function of the FBL.

Both Control and Clgalt1 Mutant follicle pairs SF when co-cultured and thus have the capacity to form MOFs due to the close interaction between follicular cells and extracellular matrix for FBL fusion; although not all co-cultured follicles did SF. Time in culture was required for follicles to SF, but this was not enough to ensure all follicles SF. We speculate this is due to differences between individual follicles. The superficial fusing of co-cultured follicles is similar to the joining of follicles in culture demonstrated by Spears group (Campbell et al. 2013) whereby cells migrate between co-cultured wildtype follicles. In Clgalt1 Mutant ovaries, we have previously observed irregularities in the theca cell layers that surround follicles (Williams and Stanley 2008; Christensen et al. 2015) and this feature may contribute to the formation of MOFs in vitro, potentially facilitating theca cells to migrate in a similar fashion (Campbell et al. 2013). To further investigate this joining, it would be interesting to investigate the levels of the different extracellular matrix proteins in the follicles, and also to determine the levels of the proteases involved in the remodelling of the FBL. It is possible that differences in these aspects are why some Mutant follicles form MOFs and some remain as SOFs. Furthermore, fully elucidating the signalling mechanism(s) to explain how the modified oocyte is directly or indirectly initiating these changes via the granulosa or theca cells is the long-term aim.
Figure 4. Confocal microscopy images of superficially fused (SF) follicles after co-culture. $(a-d)$ Serial optical sections through a SF Control follicle pair showing separation between follicles. ( $a$ $\left.d^{\prime}\right)$ Higher magnification images of the fusion point detailing separation of FBL and theca cell layers. $(e-h)$ Serial optical sections through a Clgalt1 Mutant follicle pair with indistinct space between the two follicles. $\left(e^{\prime}-h^{\prime}\right)$ Higher magnification images of the fusion point detailing FBL and theca cell layers. The border and the space between the two follicles are unclear and a breach in the FBL cannot be determined (Control mice $n=4$ and $n=4$ follicle pairs, Mutant mice $n=4$ and $n=9$ follicle pairs).
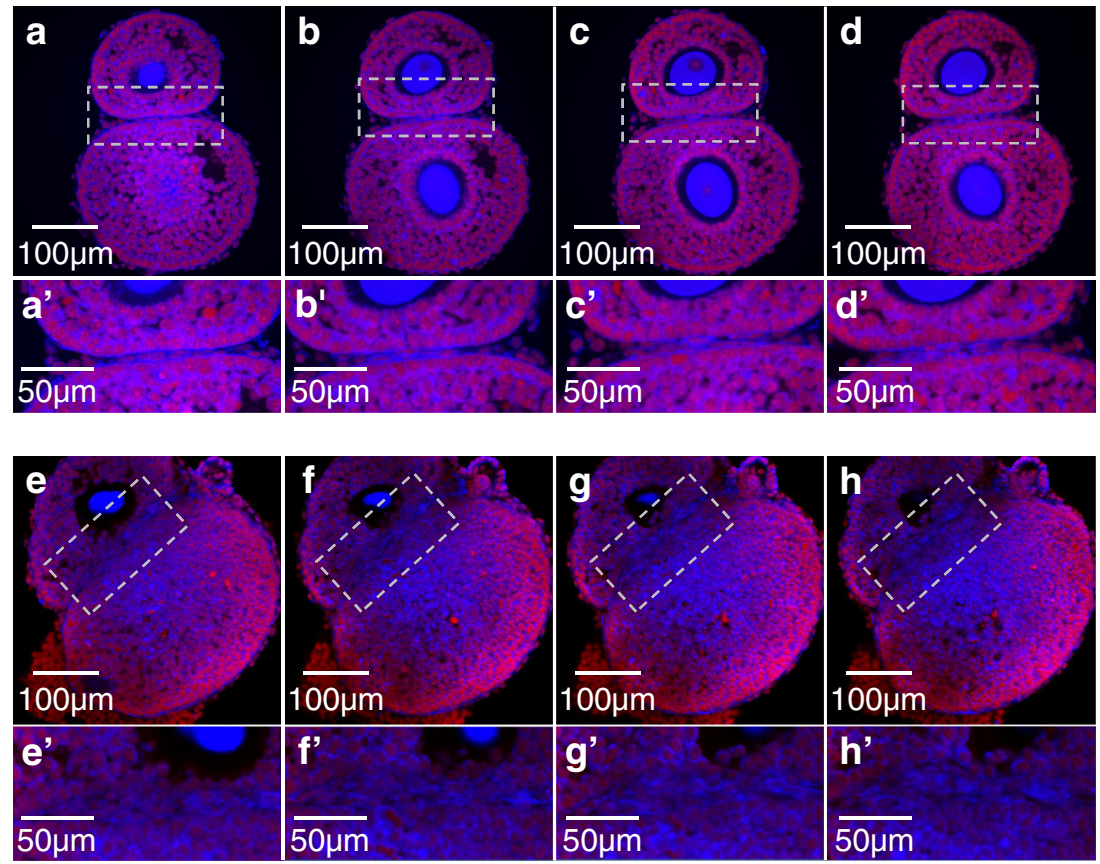
Table 1. Number of Control and Mutant MOFs formed in culture (confirmed by histological evaluation using haematoxylin stained serial sections)

\begin{tabular}{lll}
\hline & \multicolumn{2}{l}{ Number of follicle pairs } \\
\cline { 2 - 3 } & Control & Mutant \\
\hline MOF & 0 & 2 \\
SOF & 3 & 2 \\
Total & 3 & 4 \\
\hline
\end{tabular}

Co-culture of follicles has been shown to affect follicle survival although the outcome and interactions between follicles remain unclear. In our experiments, interestingly, some of the pairs grew asynchronously with one follicle larger than the other. Although follicles were of comparable size at the start of culture, it is not improbable that the follicles had different growth potentials from the outset. Indeed, the majority of follicles in an ovary do not achieve complete development. Furthermore, it is conceivable that contact or local factors from the joined follicle also affect follicle growth. Murine primary and secondary follicles cultured with other same stage follicles survive longer and grow larger than follicles cultured alone (Hornick et al. 2013). However, co-culture of two murine preantral follicles has not been shown to increase growth (Spears et al. 1996). In buffaloes, co-culture of a preantral follicle with a more developed antral follicle promoted the survival and growth of the preantral follicle (Sharma et al. 2013). Therefore, superficial fusing of follicles and the close proximity of follicles to each other may improve survival rates further. Follicle culture may provide a technique to mature oocytes from ovarian tissue stored for patients undergoing
Figure 5. Histological evaluation of SOFs and MOFs after follicle co-culture. $a-f$ Serial sections of a Control follicle pair. $\left(a^{\prime}-f^{\prime}\right)$ Higher magnification images of the area where the two Control follicles meet (black box in $(a-f)$ showing no breach in the FBL between the two follicles. $(g-h)$ Serial sections of a Clgalt1 Mutant follicle pair. (g'-l') Higher magnification images of the area where the two Clglat1 Mutant follicles meet. (black box in $g-l$ ) showing integrity of the FBL between the follicles in $\left(g^{\prime}\right)$ and $(l ')$ and a breach in the FBL $(h$ ' $\left.k^{\prime}\right)$. White arrows indicate FBL maintained its integrity. Black arrows indicate a breach in the FBL.
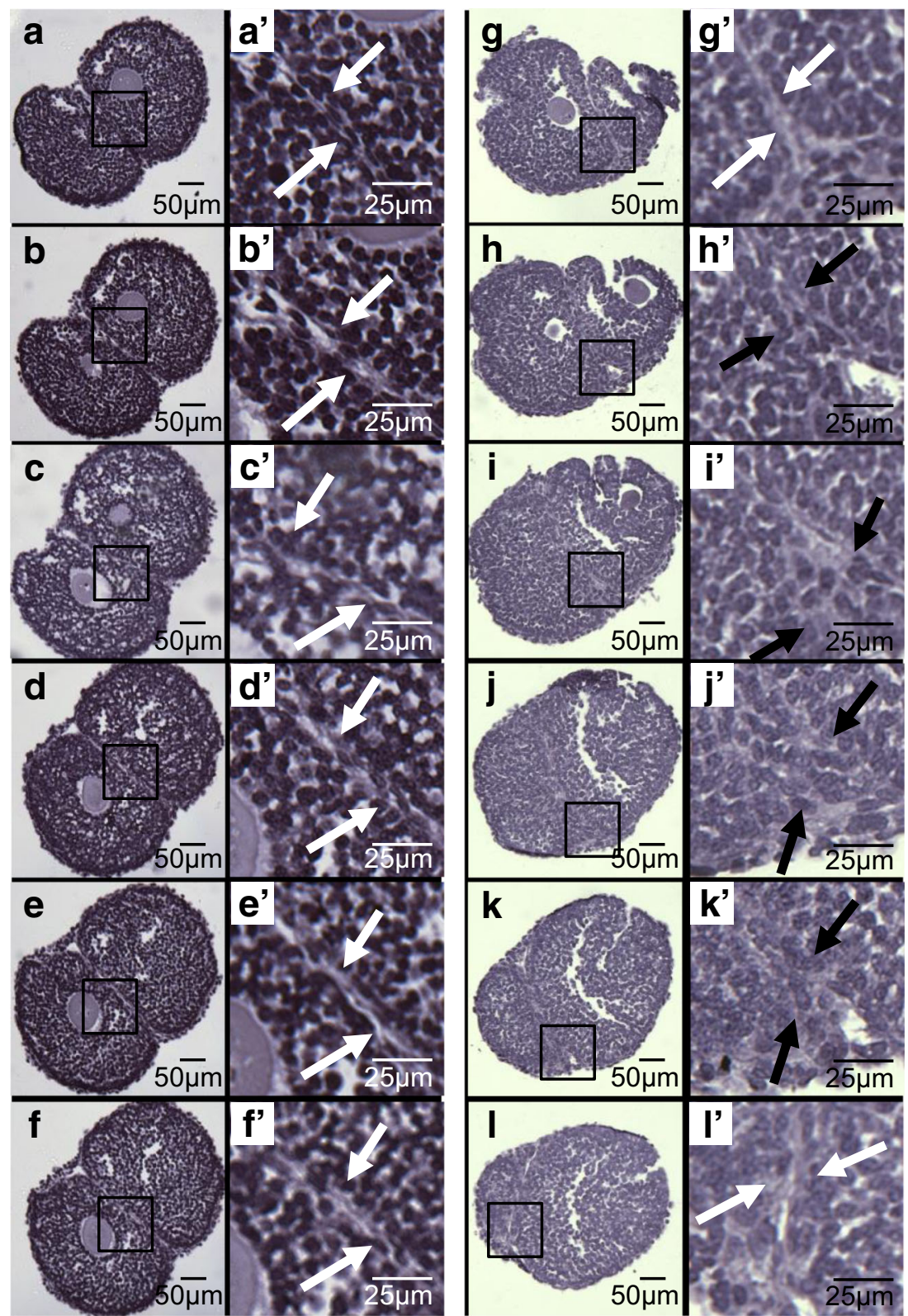
cancer therapy. Decreased apoptosis has been observed in follicles from Clgalt1 Mutant mice contributing to the phenotype of increased fertility (Grasa et al. 2015); however, there is no evidence that MOFs survive any better than SOFs.

\section{Conclusions}

If we fully comprehend the mechanisms behind how the oocyte modifies FBL generation and development enabling follicles to join, particularly in vitro, this will contribute to our understanding of follicle growth, and thus contribute to optimizing follicle development in culture for fertility treatment.

Acknowledgements The authors would like to thank Dr. S Srinivas and Dr. B Joyce for technical assistance, and Dr. J Montiel for his help with confocal imaging. This study was supported by the John Fell Fund Oxford University Press (University of Oxford, grant 073/582). H Kaune is a recipient of Becas Chile PhD scholarship from the Government of Chile and PhD scholarship for Academics from Diego Portales University, Chile.

Open Access This article is distributed under the terms of the Creative Commons Attribution 4.0 International License (http:// creativecommons.org/licenses/by/4.0/), which permits unrestricted use, distribution, and reproduction in any medium, provided you give appropriate credit to the original author(s) and the source, provide a link to the Creative Commons license, and indicate if changes were made.

\section{References}

Anderson WA, Spielman A (1971) Permeability of the ovarian follicle of Aedes aegypti mosquitoes. J Cell Biol 50:201-221

Batista F, Lu L, Williams SA, Stanley P (2012) Complex N-glycans are essential, but core 1 and 2 mucin O-glycans, O-fucose glycans, and $\mathrm{NOTCH} 1$ are dispensable, for mammalian spermatogenesis. Biol Reprod 86:179

Berkholtz CB, Lai BE, Woodruff TK, Shea LD (2006) Distribution of extracellular matrix proteins type I collagen, type IV collagen, fibronectin, and laminin in mouse folliculogenesis. Histochem Cell Biol 126:583-592

Campbell L, Trendell J, Spears N (2013) Identification of cells migrating from the thecal layer of ovarian follicles. Cell Tissue Res 353:189194

Christensen AP, Patel S, Grasa P, Christian H, Williams SA (2015) Oocyte glycoproteins regulate the form and function of the follicle basal lamina and theca cells. Dev Biol 401:287-298

Cmoch A, Groves P, Pikula S (2014) Biogenesis of invadopodia and their cellular functions. Postepy Biochem 60:62-68

Condeelis J, Segall JE (2003) Intravital imaging of cell movement in tumours. Nat Rev Cancer 3:921-930

Fein MR, Egeblad M (2013) Caught in the act: revealing the metastatic process by live imaging. Dis Model Mech 6:580-593

Grasa P, Ploutarchou P, Williams SA (2015) Oocytes lacking O-glycans alter follicle development and increase fertility by increasing follicle
FSH sensitivity, decreasing apoptosis and modifying GDF9:BMP15 expression. FASEB 29:525-539

Hayden MR, Sowers JR, Tyagi SC (2005) The central role of vascular extracellular matrix and basement membrane remodeling in metabolic syndrome and type 2 diabetes: the matrix preloaded. Cardiovasc Diabetol 4:9

Hess KA, Chen L, Larsen WJ (1998) The ovarian blood follicle barrier is both charge- and size-selective in mice. Biol Reprod 58:705-711

Hornick JE, Duncan FE, Shea LD, Woodruff TK (2013) Multiple follicle culture supports primary follicle growth through paracrine-acting signals. Reproduction 145:19-32

Irving-Rodgers HF, Morris S, Collett RA, Peura TT, Davy M, Thompson JG, Mason HD, Rodgers RJ (2009) Phenotypes of the ovarian follicular basal lamina predict developmental competence of oocytes. Hum Reprod 24:936-944

Ju T, Brewer K, D'Souza A, Cummings RD, Canfield WM (2002a) Cloning and expression of human core 1 beta1,3galactosyltransferase. J Biol Chem 277:178-186

Ju T, Cummings RD, Canfield WM (2002b) Purification, characterization, and subunit structure of rat core 1 Beta1,3galactosyltransferase. J Biol Chem 277:169-177

Pepling ME (2012) Follicular assembly: mechanisms of action. Reproduction 143:139-149

Philpott CC, Ringuette MJ, Dean J (1987) Oocyte-specific expression and developmental regulation of ZP3, the sperm receptor of the mouse zona pellucida. Dev Biol 121:568-575

Ploutarchou P, Melo P, Day AJ, Milner CM, Williams SA (2015) Molecular analysis of the cumulus matrix: insights from mice with O-glycan deficient oocytes. Reproduction 149:533-543

Rodgers RJ, Irving-Rodgers HF (2010) Morphological classification of bovine ovarian follicles. Reproduction 139:309-318

Rodgers RJ, van Wezel IL, Irving-Rodgers HF, Lavranos TC, Irvine CM, Krupa M (1999) Roles of extracellular matrix in follicular development. J Reprod Fertil Suppl 54:343-352

Sharma GT, Dubey PK, Nath A, Saikumar G (2013) Co-culture of buffalo (Bubalus bubalis) preantral follicles with antral follicles: a comparative study of developmental competence of oocytes derived from in vivo developed and in vitro cultured antral follicles. Zygote 21: 286-294

Shi S, Williams SA, Seppo A, Kurniawan H, Chen W, Ye Z, Marth JD, Stanley P (2004) Inactivation of the Mgatl gene in oocytes impairs oogenesis, but embryos lacking complex and hybrid N-glycans develop and implant. Mol Cell Biol 24:9920-9929

Siperstein MD, Unger RH, Madison LL (1968) Studies of muscle capillary basement membranes in normal subjects, diabetic, and prediabetic patients. J Clin Invest 47:1973-1999

Spears N, de Bruin JP, Gosden RG (1996) The establishment of follicular dominance in co-cultured mouse ovarian follicles. J Reprod Fertil 106:1-6

Williams SA, Stanley P (2008) Mouse fertility is enhanced by oocytespecific loss of core 1-derived O-glycans. FASEB J 22:2273-2284

Williams SA, Xia L, Cummings RD, McEver RP, Stanley P (2007) Fertilization in mouse does not require terminal galactose or Nacetylglucosamine on the zona pellucida glycans. J Cell Sci 120: 1341-1349

Yurchenco PD, Patton BL (2009) Developmental and pathogenic mechanisms of basement membrane assembly. Curr Pharm Des 15:12771294 